\title{
Robust Control of Grid-Tied Inverters using Particle Swarm Optimization and Linear Matrix Inequalities
}

\author{
Lucas C. Borin, Iury Cleveston, Gustavo G. Koch, Caio R. D. Osório, Everson Mattos, and Vinícius F. Montagner \\ Power Electronics and Control Research Group - Federal University of Santa Maria \\ Santa Maria, RS, Brazil \\ Email: lukascielo@gmail.com
}

\begin{abstract}
This paper provides a new design procedure for robust current controllers applied to LCL-filtered grid-tied inverters suitable for the integration of renewable energy sources. The design takes into account the digital implementation delay, multiple resonant controllers and operation under uncertain grid impedance. The procedure is based on the optimization of an objective function that allows to get a good trade-off between the settling of transient responses and rejection of disturbances. A particle swarm algorithm is used to find the optimal control gains and, differently from other works, here the robust stability of the closed-loop system under uncertain parameters is theoretically certified by means of linear matrix inequalities. Experimental results are shown, confirming that the closed-loop system with gains obtained by the proposed procedure presents a good tradeoff between robustness and performance, with suitable transients and grid currents with low harmonic content, complying with requirements from IEEE 1547 Standard, becoming a useful robust control design alternative for power converters in the distributed generation scenario.
\end{abstract}

Index Terms-Grid-tied inverters, Linear matrix inequalities, Particle swarm optimization, Robust control, State feedback.

\section{INTRODUCTION}

The population growth and the technological development lead to a continuous increase in the consumption of electrical energy. In order to cope with this demand in a sustainable way, renewable energy sources have become an important alternative [1], [2]. In this context, grid-tied inverters (GTIs) play a major role to interface renewable energy sources and the power grid, allowing operation with voltage, frequency and currents complying with stringent standards [3]-[5]. The control of such converters becomes more challenging when dealing with disturbances and uncertain grid impedances at the point of common coupling, which is a typical issue in the scenario of distributed generation and microgrids [6], [7].

One of the key features of GTIs is the control of the gridinjected currents, which allows to regulate the power flow between the primary source and the grid. Several current control strategies have been employed as, for instance, the proportional-integral in synchronous reference frame, proportional resonant in stationary reference frame and also state feedback [8]-[12]. In the case of GTIs with output LCL filters, there is a resonance peak that must be properly attenuated to avoid performance degradation or even instability, being active damping strategies preferred over the passive to avoid additional power losses. The design of active damping strategies becomes more difficult under grid uncertain parameters, motivating the investigation of better trade-offs between performance and robustness. In this way, metaheuristics can be an important alternative to search spaces in order to find the control gains in an optimal way, allowing to take into account practical performance indices, that can be measured even by means of simulations or based on experimental data. However, metaheuristics have not been extensively investigated for control tuning of power converters [13].

Among the metaheuristics, it is worth to highlight the particle swarm optimization (PSO), that can optimize complex functions, with simple implementation and fast execution when compared to other techniques [13], [14]. PSO has already been applied to power converters control design in [15]-[19]. A common point in these works is that robustness against uncertain parameters is not taken into account in the design stage. Moreover, since the calculations in the PSO are performed only for some parameter conditions, it may not be sufficient to ensure robust stability for the entire domain of uncertainties (which has infinite points). On the other hand, one way to represent systems subject to uncertain parameters is by means of polytopic models, from which, given a control gain, linear matrix inequalities (LMIs) can be applied to provide a theoretical certificate of stability for the entire domain of uncertainties, based on a finite number of evaluations. LMIs are recognized as a very efficient tool, being solved by specialized programs in a fast way (solution in polynomial time) [20].

The main motivation for the present work is to provide a design procedure that combines PSO and LMIs, leading to control gains that ensure suitable grid currents even under disturbances, parameter uncertainties and delay from digital control. The proposed procedure is able to find state feedback control gains in a given hyper-rectangle, thanks to the PSO, and also can certify the robust stability of the closed-loop system under uncertain grid impedance, thanks to the LMIs. The optimization which guides the control design is based on a novel objective function, which takes into account a limit for the closed-loop poles and also a limit for the transmission of disturbances from the control input to the system output. Experimental results are presented and confirm the effectiveness of the proposed procedure for grid-current control. 


\section{State Space Model with Uncertainties}

A three-phase inverter connected to the grid by means of an LCL filter is shown in Figure 1. The grid is assumed as predominately inductive, and the equivalent grid inductance at the point of common coupling (PCC) is given by the uncertain parameter $L_{g r}$.

From the inverter output voltages, considering a balanced system and that there is no path for the current ' 0 ', a state space model of the plant in $\alpha \beta 0$ stationary reference frame can be written as (see, for instance, [21] for details)

$$
\begin{gathered}
{\left[\begin{array}{c}
\dot{x}_{\alpha} \\
\dot{x}_{\beta}
\end{array}\right]=\left[\begin{array}{cc}
A\left(L_{g}\right) & 0 \\
0 & A\left(L_{g}\right)
\end{array}\right]\left[\begin{array}{c}
x_{\alpha} \\
x_{\beta}
\end{array}\right]+} \\
{\left[\begin{array}{cc}
B_{u} & 0 \\
0 & B_{u}
\end{array}\right]\left[\begin{array}{l}
u_{\alpha} \\
u_{\beta}
\end{array}\right]+\left[\begin{array}{cc}
B_{w}\left(L_{g}\right) & 0 \\
0 & B_{w}\left(L_{g}\right)
\end{array}\right]\left[\begin{array}{l}
v_{g \alpha} \\
v_{g \beta}
\end{array}\right]}
\end{gathered}
$$

where $x_{\alpha}$ and $x_{\beta}$ are state vectors, $u_{\alpha}$ and $u_{\beta}$ are control inputs, $v_{g \alpha}$ and $v_{g \beta}$ are disturbance inputs, and the uncertain parameter $L_{g}$ is given by

$$
L_{g}=L_{c 2}+L_{g r}
$$

due to the uncertainty on $L_{g r}$.

Notice that (1) represents two single-phase decoupled systems. For instance, for axis $\alpha$, this model is given by

$$
\dot{x}_{\alpha}=A\left(L_{g}\right) x_{\alpha}+B_{u} u_{\alpha}+B_{w}\left(L_{g}\right) v_{g \alpha}
$$

being

$$
\begin{aligned}
& A\left(L_{g}\right)=\left[\begin{array}{ccc}
0 & -\frac{1}{L_{c 1}} & 0 \\
\frac{1}{C_{f}} & 0 & -\frac{1}{C_{f}} \\
0 & \frac{1}{L_{g}} & 0
\end{array}\right], B_{u}=\left[\begin{array}{c}
\frac{1}{L_{c 1}} \\
0 \\
0
\end{array}\right], \\
& B_{w}\left(L_{g}\right)=\left[\begin{array}{c}
0 \\
0 \\
-\frac{1}{L_{g}}
\end{array}\right], x_{\alpha}=\left[\begin{array}{c}
i_{c \alpha} \\
v_{c \alpha} \\
i_{g \alpha}
\end{array}\right]
\end{aligned}
$$

where $i_{c \alpha}$ is the current in the inductor on the converter side, $v_{c \alpha}$ is the voltage in the filter capacitor and $i_{g \alpha}$ is the current in the inductor on the grid side, for axis $\alpha$.

Since a model identical to (3)-(4) can be used for axis $\beta$, the subscripts $\alpha$ and $\beta$ are suppressed from now on.

The parametric uncertainty on $L_{g}$ can be taken into account

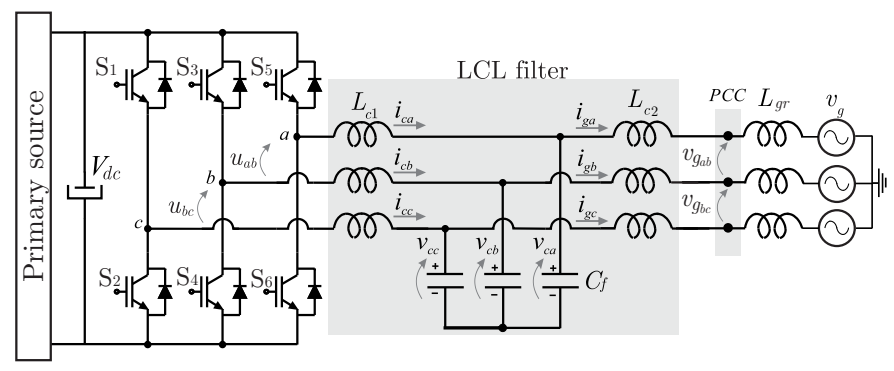

Figure 1. Three-phase grid connected inverter through LCL filter. by means of the polytopic representation

$$
\dot{x}=A(\theta) x+B_{u} u+B_{w}(\theta) v_{g}
$$

where

$$
A(\theta)=\theta A_{1}+(1-\theta) A_{2}, \quad B_{w}(\theta)=\theta B_{w 1}+(1-\theta) B_{w 2}
$$

and $\theta$ is a real parameter such that $0 \leq \theta \leq 1$.

In this representation, for instance, $A_{1}$ and $A_{2}$ are the vertices of the polytope, obtained by evaluating $A\left(L_{g}\right)$ for the minimum and maximum values of $L_{g}$.

For the application of a digital control strategy, consider the plant discretization with a sufficiently small sampling period $T_{s}$, leading to the discrete polytopic model

$$
\begin{aligned}
& x(n+1)=A_{d}(\theta) x(n)+B_{u d}(\theta) \varphi(n)+B_{w d}(\theta) v_{g}(n) \\
& y(n)=C x(n)=\left[\begin{array}{lll}
0 & 0 & 1
\end{array}\right]\left[i_{c}(n) v_{c}(n) i_{g}(n)\right]^{\prime}
\end{aligned}
$$

where

$$
A_{d}(\theta)=\theta A_{d 1}+(1-\theta) A_{d 2}, \quad A_{d i}=e^{A_{i} T_{s}}
$$

$$
B_{u d}(\theta)=\theta B_{u d 1}+(1-\theta) B_{u d 2}, B_{u d i}=\int_{0}^{T_{s}} e^{A_{i} \tau} B_{u i} d \tau
$$

$B_{w d}(\theta)=\theta B_{w d 1}+(1-\theta) B_{w d 2}, B_{w d i}=\int_{0}^{T_{s}} e^{A_{i} \tau} B_{w i} d \tau$

with $i=1$ or $i=2$, representing each one of the vertices.

The additional state

$$
\varphi(n+1)=u(n)
$$

is included to represent the transport delay in the control signal implementation [22].

Based on the internal model principle [23], to guarantee tracking of sinusoidal references and the rejection of harmonic disturbances, resonant controllers can be employed, being represented, in the continuous case, by

$$
\left[\begin{array}{c}
\dot{\delta_{r}} \\
\ddot{\delta}_{r}
\end{array}\right]=\overbrace{\left[\begin{array}{cc}
0 & 1 \\
-\omega_{n}^{2} & -2 \xi \omega_{n}
\end{array}\right]}^{R_{c}}\left[\begin{array}{c}
\delta_{c} \\
\dot{\delta}_{c}
\end{array}\right]+\overbrace{\left[\begin{array}{l}
0 \\
1
\end{array}\right]}^{T_{c}} e
$$

where $\omega_{n}$ is the resonant frequency and $\xi$ is the damping factor.

A discrete-time model of the resonant controller can be writen as [22]

$$
x_{r}(n+1)=R_{d} x_{r}(n)+T_{d} e(n)
$$

with

$$
R_{d}=e^{R_{c} T s}, \quad T_{d}=\int_{0}^{T_{s}} e^{R_{c} \tau} T_{c} d \tau
$$

Generalizing, for $\ell$ independent resonant controllers, one has

$$
R=\left[\begin{array}{cccc}
R_{d 1} & 0 & 0 & 0 \\
0 & R_{d 2} & 0 & 0 \\
0 & 0 & \ddots & 0 \\
0 & 0 & 0 & R_{d \ell}
\end{array}\right] \quad T=\left[\begin{array}{c}
T_{d 1} \\
T_{d 2} \\
\vdots \\
T_{d \ell}
\end{array}\right]
$$


Finally, the augmented model can be written as

$$
\begin{gathered}
\rho(n+1)=A_{\text {aug }}(\theta) \rho(n)+B_{\text {uaug }} u(n) \\
+B_{\text {waug }}(\theta) v_{g}(n)+B_{\text {raug }} r(n) \\
y(n)=C_{\text {aug }} \rho(n)
\end{gathered}
$$

where $\rho(n)=\left[x(n)^{\prime} \varphi(n) x_{r}(n)^{\prime}\right]^{\prime}$ is the augmented state vector and

$$
\begin{aligned}
& A_{\text {aug }}(\theta)=\left[\begin{array}{ccc}
A_{d}(\theta) & B_{u d}(\theta) & 0 \\
0 & 0 & 0 \\
-T C & 0 & R
\end{array}\right], B_{\text {uaug }}=\left[\begin{array}{c}
0 \\
1 \\
0
\end{array}\right] \\
& B_{\text {waug }}(\theta)=\left[\begin{array}{c}
B_{w d}(\theta) \\
0 \\
0
\end{array}\right], B_{\text {raug }}=\left[\begin{array}{c}
0 \\
0 \\
T
\end{array}\right]
\end{aligned}
$$

This model is useful for the design of state feedback controller, and will be employed in the next section together with the PSO.

\section{Control Design Procedure}

Given the state space formulation of the problem in Section II, one can use the state feedback control law [22]

$$
u(n)=K \rho(n)=\left[\begin{array}{lll}
K_{x} & K_{\varphi} & K_{r}
\end{array}\right]\left[\begin{array}{c}
x(n) \\
\varphi(n) \\
x_{r}(n)
\end{array}\right]
$$

where $K$ is the vector of control gains.

In the proposed design procedure, the control gains will be obtained by means of a PSO algorithm, which requires an objective function to guide the optimization.

\section{A. Objective function}

The objective function has the purpose of measuring the system performance for a given control gain $K$. In the context of grid-tied inverters, the problem is to design robust control gains capable to ensure that the closed-loop grid currents track sinusoidal references even under disturbances and parameter uncertainties.

In order to achieve that, consider the proposed objective function

$$
F(K)=\sigma(K) \gamma(K)+\frac{(380 \sigma(K)-360)}{1+e^{-1000 \sigma(K)+1000}}
$$

In (19), the function $\sigma(K)$ provides a stability index evaluating the maximum module of the closed-loop system eigenvalues, defined by the maximum value of

$$
\max \left|\lambda\left(A_{\text {aug }}(\theta)+B_{\text {uaug }} K\right)\right|
$$

evaluated for $\theta=0$ and $\theta=1$, i.e., for the extreme values of $L_{g}$. It is known that evaluating the stability only at the vertices of a polytope is a necessary (but not sufficient) condition for the stability of the entire domain. However, it is used here since, from the computational point of view, this provides a simplified and fast way to approach the stability, which will be theoretically guaranteed by the LMI tests in the next section.
The function $\gamma(K)$ is a measure of the harmonic attenuation from the output PWM voltages to the current $i_{g}$, defined by the maximum value of

$$
\max \left|C_{\text {aug }}\left(e^{j \omega T_{s}} I-\left(A_{\text {aug }}(\theta)+B_{\text {uaug }} K\right)\right)^{-1} B_{\text {uaug }}\right|
$$

evaluated for $\theta=0$ and $\theta=1$, over the frequency range $\omega \in$ $\left[0, \frac{2 \pi}{T_{s}}\right]$. These harmonics can be related with nonlinearities, as dead-time in the driving of the inverter switches, for instance.

Concerning the objective function, the first term of (19) allows to get a good trade-off between (20) and (21). However, evaluate only the first term may not guarantee that the solution is stable (i.e., $\sigma<1$ ). For this, a second term is included to play the role of a penalization, using the sigmoid function. Thus, for unstable solutions, the value of the sigmoid function increases the objective function for higher values of $\sigma$. On the other hand, for values of $\sigma<1$, the sigmoid function has almost zero value. In addition, the angular coefficient (equals to 380) and linear coefficient (equals to 360) are designed to increase the convergence of the solutions to the stable region.

The problem now is to find the robust control gain $K^{\star}$ that solves the following optimization:

$$
K^{\star}=\arg \min _{K \in \mathcal{K}} F(K)
$$

where $F(K)$ is given by (19), taking into account different controller candidates belonging to a search space $\mathcal{K}$, and (22) must be evaluated for $\theta=0$ and $\theta=1$.

The following subsection presents the PSO algorithm applied to the problem of finding robust control gains.

\section{B. Particle swarm optimization}

In order to use the PSO [14] in the control design problem above, consider that each vector $K$ in (18) defines a particle position $s_{j}$ (i.e., a point in a search space) with $D$ dimensions, given by

$$
s_{j}=\left[\begin{array}{lllll}
s_{j, 1} & s_{j, 2} & s_{j, 3} & \cdots & s_{j, D}
\end{array}\right]
$$

The algorithm initially creates a swarm of particles, randomly distributed in the search space. By evaluating the objective function, a fitness value is associated to the position of each particle. In successive iterations (called epochs) the positions are updated, and the swarm moves in the search space in order to find the minimum of the objective function.

For a given epoch $m$, a recursive velocity equation is used to update the position of each particle. The best position that each particle has ever obtained $\left(P_{j . b e s t}\right)$, and the best position among all particles $\left(G_{b e s t}\right)$ are defined by the objective function and the values are stored. The velocity and position recursive equations are given, respectively, by

$$
\begin{gathered}
v_{j}^{m+1}=\omega v_{j}^{m}+\eta_{1} r_{1}\left(P_{j . b e s t}-s_{j}^{m}\right)+\eta_{2} r_{2}\left(G_{\text {best }}-s_{j}^{m}\right) \\
s_{j}^{m+1}=s_{j}^{m}+v_{j}^{m+1}
\end{gathered}
$$

being $\eta_{1}$ e $\eta_{2}$ the cognitive and social coefficients, respectivelly, $r_{1}$ and $r_{2}$ random values between $[0,1]$ and $\omega$ the inertia factor that decelerates the particles during the execution, for a better convergence. 
The PSO execution used here has the following steps:

1) configure the PSO parameters;

2) initialize the particles randomly in the search space;

3) calculate the objective function for each particle;

4) update $P_{j . b e s t}$ and $G_{\text {best }}$;

5) update position and velocity of each particle;

$6)$ if the stop criterion is reached, inform the best particle $\left(G_{\text {best }}\right)$ and end the execution. If not, return to step 2.

At the end of the execution, the PSO algorithm returns the particle (i.e., the control gain $K^{\star}$ ) with the lowest objective function value associated $\left(G_{b e s t}\right)$. The number of particles, the number of epochs, and the coefficients $\eta_{1}$ and $\eta_{2}$ are set in order to ensure convergence of the objective function with viable computational effort.

After the PSO execution, a theoretical certificate of robust stability is provided, by means of LMIs, as can be seen in the next section.

\section{Robust Stability By MEANS OF LMIS}

The control gain $K^{\star}$ provided by the PSO is obtained evaluating the closed-loop system stability and performance only for the vertices of the polytopic model. In order to provide a theoretical certificate of robust stability valid for the entire polytope, LMI based tests can be used, as follows.

The closed-loop system

$$
\rho(n+1)=G(\theta) \rho(n)
$$

with

$$
G(\theta)=A_{\text {aug }}(\theta)+B_{\text {uaug }}(\theta) K
$$

is stable for all $0 \leq \theta \leq 1$ if there exist symmetric positive matrices $P_{1}$ and $P_{2}$ such that

$$
G_{1}^{\prime} P_{1} G_{1}-P_{1}<-I, \quad G_{2}^{\prime} P_{2} G_{2}-P_{2}<-I
$$

and

$$
\begin{aligned}
& G_{1}^{\prime} P_{1} G_{2}+G_{2}^{\prime} P_{1} G_{1}+G_{1}^{\prime} P_{2} G_{1}-2 P_{1}-P_{2}<I, \\
& G_{2}^{\prime} P_{2} G_{1}+G_{1}^{\prime} P_{2} G_{2}+G_{2}^{\prime} P_{1} G_{2}-2 P_{2}-P_{1}<I
\end{aligned}
$$

The proof can be found in [24] for the general case of $N$ vertices, and was specialized here for 2 vertices. The results with the above LMIs are less conservative than the well known quadratic stability [20], allowing to get theoretical certificates of robust stability even when the quadratic stability fails.

\section{CASE Study and Experimental VALidation}

As a case study, consider the GTI shown in Figure 1, with parameters given in Table I. Note that there are 4 resonant controllers, which lead to a $x_{r}$ vector with 8 states, that, together with the 3 states of $x$ and the state $\phi$, results in an augmented state vector of 12 states.

The PSO parameters are chosen as: 50 particles, 200 epochs and $\eta_{1}=\eta_{2}=0.5$. For each entry of $K$, the limits that defines the search space are given in Table II. These values were obtained from a digital linear quadratic regulator control vector, tuned with $Q$ being the identity matrix and $R=1$.
Table I

GTI PARAMETERS.

\begin{tabular}{c|c}
\hline \hline Parameters & Values \\
\hline$L_{c 1}, C_{f}$ and $L_{c 2}$ & $1 \mathrm{mH}, 62 \mu \mathrm{F}$ and $0.3 \mathrm{mH}$ \\
$L_{g}$ & {$[0,1] \mathrm{mH}$} \\
DC-link voltage $V_{d c}$ & $400 \mathrm{~V}$ \\
Grid voltage $v_{g}$ & $180 \mathrm{~V}$ (peak);60 Hz \\
Sampling frequency & $20040 \mathrm{~Hz}$ \\
Switching frequency & $10020 \mathrm{~Hz}$ \\
Frequencies of resonant controllers & $60,180,300$ and $420 \mathrm{~Hz}$ \\
Damping factor & 0.0001 \\
\hline \hline
\end{tabular}

Table II

SearCh SPACE $\mathcal{K}$ Limits FOR the GCC CASE STUdy.

\begin{tabular}{c|c|c|c|c|c|c|c|c|c|c|c|c}
\hline \hline$s_{j}$ & $s_{j, 1}$ & $s_{j, 2}$ & $s_{j, 3}$ & $s_{j, 4}$ & $s_{j, 5}$ & $s_{j, 6}$ & $s_{j, 7}$ & $s_{j, 8}$ & $s_{j, 9}$ & $s_{j, 10}$ & $s_{j, 11}$ & $s_{j, 12}$ \\
\hline$s_{j \min }$ & -15 & -15 & -15 & -15 & 0 & -100 & 0 & -50 & 0 & -50 & 0 & -50 \\
$s_{j \max }$ & 0 & 0 & 0 & 0 & 100 & 0 & 50 & 0 & 50 & 0 & 50 & 0 \\
\hline \hline
\end{tabular}

The PSO was executed several times, always converging to viable controllers. After a typical execution, the PSO led to the solution

$$
\begin{aligned}
& K^{\star}=\left[\begin{array}{llll}
-9.6783 & -2.1732-1.2914-0.2858
\end{array}\right. \\
& 73.7784-73.745718 .1096-17.6463 \\
& 23.0582-23.458723 .0796-23.8390]
\end{aligned}
$$

for which the fitness has the evolution shown in Figure 2(a).

Evaluating functions (20) and (21) for the gain (30) one has $\sigma=0.9933$ and $\gamma=0.3109$. The value of $\sigma$ can be confirmed by the eigenvalues location in the unit circle, shown in Figure 2(b), for a sweep in $L_{g}$ from $0 \mathrm{mH}$ to $1 \mathrm{mH}$. The value of $\gamma$ can be confirmed by the frequency response of model (16) from the input $u$ to the output $i_{g}$, shown in Figure 3, confirming rejection of disturbances for all frequencies, specially in the harmonics of the resonant controller, which also ensure good rejection of harmonics from the grid voltages, which was confirmed by time-simulations.

When verifying the feasibility of the LMIs (28) and (29) with the control gain (30), the LMI Control Toolbox from Matlab confirm that there exist symmetric positive matrices $P_{1}$ and $P_{2}$ solving the problem, which theoretically certifies the stability for the entire domain of uncertain parameters (i.e., for $L_{g}$ assuming any value from $L_{g \min }$ to $L_{g \max }$ ).

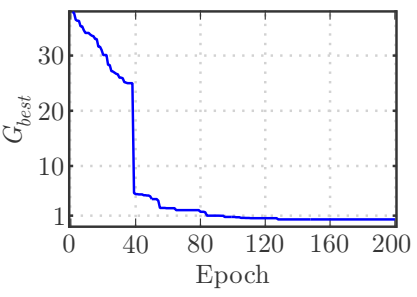

(a)

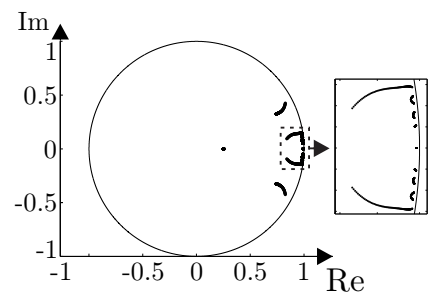

(b)
Figure 2. (a) Evolution of $G_{\text {best }}$ in each epoch; (b) Closed-loop eigenvalues for a sweep in $L_{g}$, confirming the value $\sigma=0.9933$. 


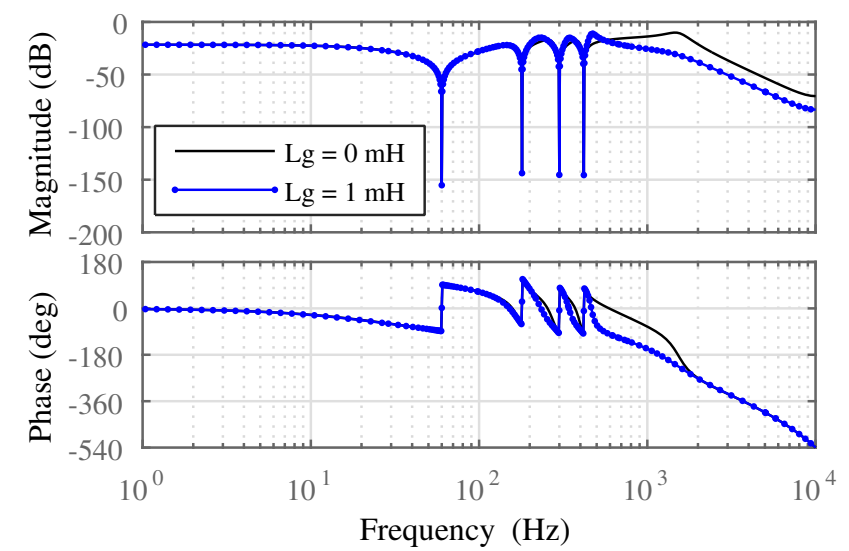

Figure 3. Frequency response from $u$ to $i g$, for the extreme values of $L_{g}$, confirming the value $\gamma=0.3109$ (i.e. $-10.15 \mathrm{~dB}$ ).

\section{A. Experimental Results}

To evaluate the system performance with control gains in (30), experimental results are obtained with a $5.4 \mathrm{~kW}$ prototype. The prototype is comprised by a three-phase inverter based on IGBTs and a three-phase LCL filter, with system parameters in Table I. Filter states (currents and voltages) are measured using Hall effect sensors. The control law is synthesized in a DSP TMS320F28335 and the grid synchronization with the PCC is provided by a Kalman filter algorithm [25].

Figure 4 shows the grid-injected currents, in $\alpha$ and $\beta$ axes, under reference amplitude variations for the converter connected to a real utility grid. The first variation represents the start-up of the system, injecting active power into the grid, while the second one represents an increase in the current amplitude to $20 \mathrm{~A}$. From these results it is possible to verify that the system is capable of tracking sinusoidal grid-current references with suitable steady-state and transient responses.

The three-phase currents related to the test performed in

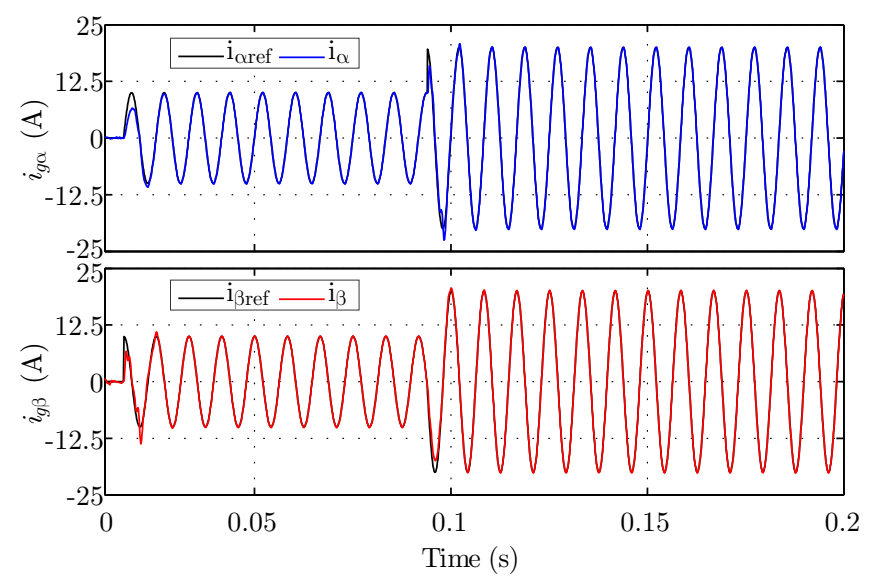

Figure 4. Experimental grid-injected currents in $\alpha$ axis (top) and $\beta$ axis (bottom) for an experimental test under reference variations (data from DSP).

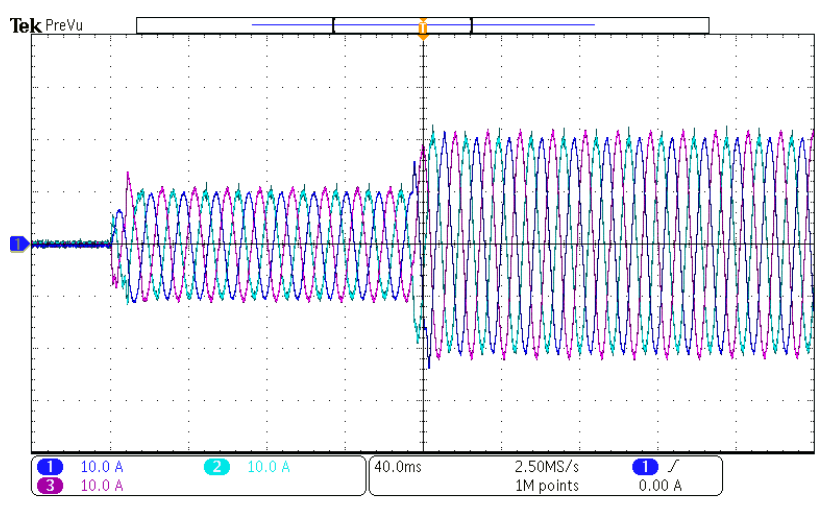

(a)

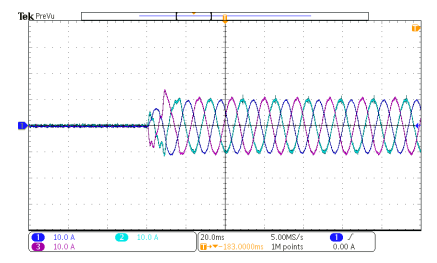

(b)

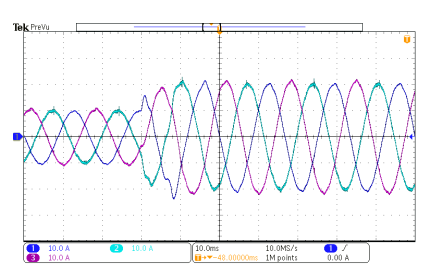

(c)
Figure 5. Experimental three-phase grid currents for the reference variations in Figure 4 (vertical scale: $10 \mathrm{~A} / \mathrm{div}$ ): (a) full test; (b) converter start-up; (c) increase in the active power.

Figure 4 are shown in Figure 5 (a), and the transient responses are detailed in Figure 5 (b) and (c), highlighting the overall good closed-loop performance.

Figure 6 (a) shows the three-phase grid currents in steadystate. For one of the phases, the harmonic spectrum is given in Figure 6 (b), showing that the closed-loop system with control gain (30) is able to properly synthesize grid currents with individual harmonic components that comply with the limits from the IEEE 1547 Standard, and with total harmonic distortion less than $5 \%$, also complying with this Standard.

Finally, to evaluate the closed-loop stability under uncertain grid inductances, a test is performed with the filter disconnected of the PCC. A short-circuit is then applied to the output of the filter, and the grid inductance is emulated with additional inductors, included in series with $L_{c 2}$. Figure 7 (a) and (b) shows the system start-up with $L_{g m i n}$ (i.e. $L_{g}=0 \mathrm{mH}$ ) and $L_{\text {gmax }}$ (i.e. $L_{g}=1 \mathrm{mH}$ ), respectively. It is possible to confirm

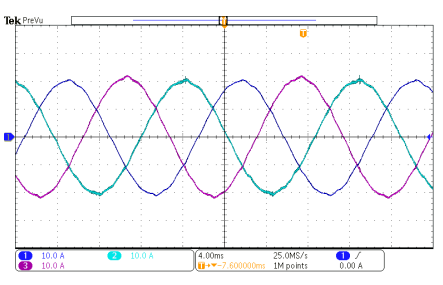

(a)

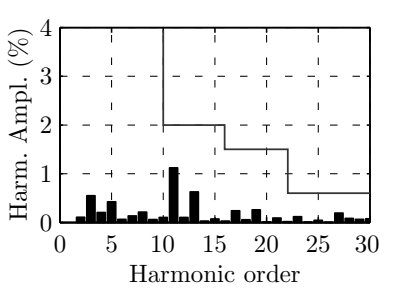

(b)
Figure 6. Experimental results for harmonic content analyses (vertical scale: $10 \mathrm{~A} / \mathrm{div}$ ): (a) Steady-state currents; (b) harmonic spectrum and limits from IEEE 1547 Standard. 


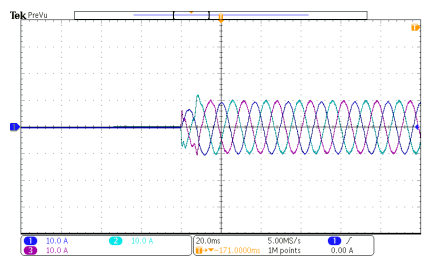

(a)

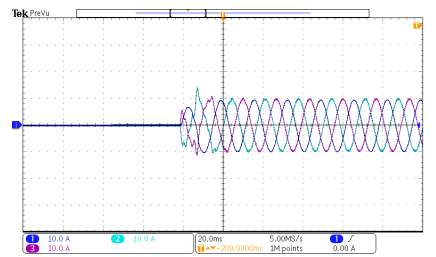

(b)
Figure 7. Experimental results for stability evaluation under uncertain parameter (vertical scale: $10 \mathrm{~A} / \mathrm{div}$ ): three-phase grid currents for an off-grid test emulating (a) $L_{g \min }$ and (b) $L_{g \max }$.

the stability under uncertain parameters, ensured by the LMIs, with suitable transient and steady-state responses.

\section{ACKNOWLEDGMENTS}

This study was financed in part by the Coordenação de Aperfeiçoamento de Pessoal de Nível Superior - Brasil (CAPES/PROEX) - Finance Code 001. The authors would also like to thank the INCT-GD and the finance agencies (CNPq 465640/2014-1, CNPq 309536/2018-9, CAPES 23038.000776/2017-54 and FAPERGS 17/2551-0000517-1).

\section{CONCLUSION}

This paper provides an automatic procedure for the design of robust state feedback current controllers for GTIs, leading to grid-injected currents in compliance with requirements from IEEE 1547 Standard. The design is based on a PSO, which searches the control gains guided by the minimization of an objective function that takes into account a trade-off between the settling of transient responses and disturbance rejection. Differently of other papers, LMIs are used to theoretically certify the robust stability of the closed-loop for the entire interval of grid uncertain inductances. Experimental results are shown, confirming compliance of the steady-state responses with requirements from IEEE 1547 Standard for individual harmonics and total harmonic distortion, and also suitable transient recovers.

\section{REFERENCES}

[1] R. Teodorescu, M. Liserre, and P. Rodríguez, Grid Converters for Photovoltaic and Wind Power Systems, ser. Wiley - IEEE. John Wiley \& Sons, 2011.

[2] F. Blaabjerg and K. Ma, "Future on power electronics for wind turbine systems," IEEE Journal of Emerging and Selected Topics in Power Electronics, vol. 1, no. 3, pp. 139-152, Sep. 2013.

[3] F. Blaabjerg, R. Teodorescu, M. Liserre, and A. Timbus, "Overview of control and grid synchronization for distributed power generation systems," IEEE Transactions on Industrial Electronics, vol. 53, no. 5, pp. $1398-1409$, oct. 2006.

[4] C. Poongothai and K. Vasudevan, "Design oflclfilter for grid-interfaced pv system based on cost minimization," IEEE Transactions on Industry Applications, vol. 55, no. 1, pp. 584-592, Jan 2019.

[5] IEEE:1547 Standard for interconnecting distributed resources with electric power systems, Institute of Electrical and Electronics Engineers Std., 2011.

[6] I. Gabe, V. F. Montagner, and H. Pinheiro, "Design and implementation of a robust current controller for VSI connected to the grid through an LCL filter," IEEE Transactions on Power Electronics, vol. 24, no. 6, pp. 1444-1452, June 2009.
[7] M. Lu, A. Al-Durra, S. M. Muyeen, S. Leng, P. C. Loh, and F. Blaabjerg, "Benchmarking of stability and robustness against grid impedance variation forlcl-filtered grid-interfacing inverters," IEEE Transactions on Power Electronics, vol. 33, no. 10, pp. 9033-9046, Oct 2018.

[8] J. Dannehl, F. W. Fuchs, S. Hansen, and P. B. Thogersen, "Investigation of active damping approaches for PI-based current control of gridconnected pulse width modulation converters with LCL filters," IEEE Transactions on Industry Applications, vol. 46, no. 4, pp. 1509-1517, July 2010.

[9] R. Teodorescu, F. Blaabjerg, M. Liserre, and P. Loh, "Proportionalresonant controllers and filters for grid-connected voltage-source converters," IEE Proceedings Electric Power Applications, vol. 153, no. 5, pp. 750-762, September 2006.

[10] L. A. Maccari, Jr., J. R. Massing, L. Schuch, C. Rech, H. Pinheiro, R. C. L. F. Oliveira, and V. F. Montagner, "LMI-based control for gridconnected converters with LCL filters under uncertain parameters," IEEE Transactions on Power Electronics, vol. 29, no. 7, pp. 3776-3785, July 2014.

[11] G. G. Koch, L. A. Maccari, R. Oliveira, and V. F. Montagner, "Robust $\mathcal{H}_{\infty}$ state feedback controllers based on lmis applied to grid-connected converters," IEEE Transactions on Industrial Electronics, pp. 1-1, 2018.

[12] C. R. D. Osório, G. G. Koch, H. Pinheiro, R. C. L. F. Oliveira, and V. F. Montagner, "Robust current control of grid-tied inverters affected by lcl filter soft-saturation," IEEE Transactions on Industrial Electronics, pp. 1-1, 2019.

[13] S. E. De León-Aldaco, H. Calleja, and J. A. Alquicira, "Metaheuristic optimization methods applied to power converters: A review," IEEE Transactions on Power Electronics, vol. 30, no. 12, pp. 6791-6803, 2015.

[14] R. Eberhart and J. Kennedy, "A new optimizer using particle swarm theory," in Micro Machine and Human Science, 1995. MHS'95., Proceedings of the Sixth International Symposium on. IEEE, 1995, pp. 39-43.

[15] B. Ufnalski, A. Kaszewski, and L. M. Grzesiak, "Particle swarm optimization of the multioscillatory LQR for a three-phase four-wire voltage-source inverter with an LC output filter," IEEE Transactions on Industrial Electronics, vol. 62, no. 1, pp. 484-493, 2015.

[16] A. Murari, L. Rodrigues, J. Altuna, A. S. Potts, L. Almeira, and A. Sguarezi Filho, "A LQRI power control for DFIG tuned by a weighted-PSO," Control Engineering Practice, vol. 85, pp. 41-49, 2019.

[17] A. Zobaa and A. Lecci, "Particle swarm optimisation of resonant controller parameters for power converters," IET power electronics, vol. 4, no. 2, pp. 235-241, 2011.

[18] S. S. Sebtahmadi, H. B. Azad, S. H. A. Kaboli, M. D. Islam, and S. Mekhilef, "A PSO-DQ current control scheme for performance enhancement of Z-source matrix converter to drive IM fed by abnormal voltage," IEEE Transactions on Power Electronics, vol. 33, no. 2, pp. 1666-1681, 2017.

[19] Z.-L. Gaing, "A particle swarm optimization approach for optimum design of PID controller in AVR system," IEEE transactions on energy conversion, vol. 19, no. 2, pp. 384-391, 2004.

[20] S. Boyd, L. El Ghaoui, E. Feron, and V. Balakrishnan, Linear Matrix Inequalities in System and Control Theory. Philadelphia, PA: SIAM Studies in Applied Mathematics, 1994.

[21] L. A. Maccari, A. C. L. Santini, H. Pinheiro, R. C. L. F. de Oliveira, and V. F. Montagner, "Robust optimal current control for grid-connected three-phase pulse-width modulated converters," IET, Power Electronics, vol. 8, no. 8, pp. 1490-1499, 2015.

[22] K. Ogata, Discrete-time control systems. Prentice Hall, 1995.

[23] B. A. Francis, A Course in $\mathcal{H}_{\infty}$ Control Theory, ser. Lectures Notes in Control and Information Sciences. New York: Springer-Verlag, 1987, vol. 88.

[24] D. C. W. Ramos and P. L. D. Peres, "A less conservative LMI condition for the robust stability of discrete-time uncertain systems," Systems \& Control Letters, vol. 43, no. 5, pp. 371-378, August 2001.

[25] R. Cardoso, R. F. de Camargo, H. Pinheiro, and H. A. Gründling, "Kalman filter based synchronisation methods," Generation, Transmission Distribution, IET, vol. 2, no. 4, pp. 542-555, july 2008. 